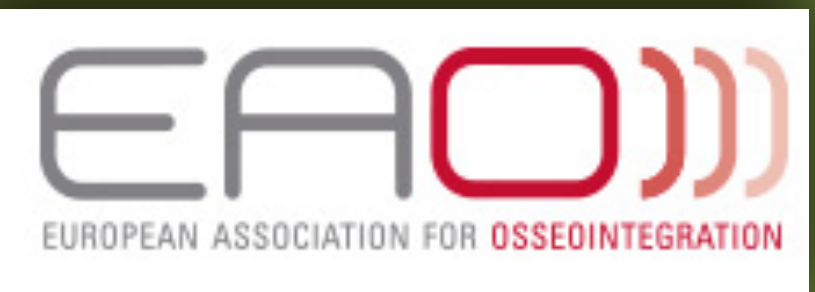

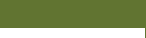

IMPLANT THERAPY

OUTCOMES, SURGICAL ASPECTS

\section{The aid of a Dynamic Computer Guided Surgery System in cases of atrophic maxilla.}

Dr.Pierluigi Pelagalli/Dr.Giulia Arcioni

Studio dentistico Dr.Pierluigi Pelagalli.

\section{Abstract}

The aim of that work is to show how the dynamic navigation system Navident improve the results of our surgeries especially in cases of atrophic maxila.

We present the methodology showing two case of atrophic maxilla that we considered achievable with the aid of the computer guided surgery. In both cases the implants position was respected like we virtual planned on the software.We were able to realize a precise and safe surgery respecting the anatomic structures.

We consider the dynamic navigation system an important aid for the surgeon to realize a safety,functional and precise rehabilitation.

\section{Background and Aim}

The aim of implant surgery is to insert implants in the right prosthetic position. Often patient's anatomy tends to foreclose this goal. Actually we have different computer aid system to plan the implant surgery respecting patient's anatomy and prosthetic project. Computer guided surgery has the limit of the need of a surgical guide that does not allow intraoperative modifications of the planning, a complex management of the soft tissues and the demand of a specific drill system. The needs of a minimum mouth's opening by the patient, due to the stent's size and the bushings, often precluding the use in the posterior area. In cases showed below we used a dynamic computer guided surgery system. This technique doesn't need a surgical template. The surgeon works freehand guided, during the implant surgery, by optical sensors. This sensor, attached to both the patient (jaw tag) and surgical handpiece (drill tag), transmit the three dimensional position by a camera that show us in to the display the exact position of the drill during implant site preparation (Fig 1). So the system guided us to insert implants in the same position that we planned.

In this work we present different clinical case in which the anatomic conditions made complex the correct angulation of the implants. It was possible to insert the implants in the ideal prosthetic position with the best angulation related to the available bone.

The aim of that work is to show how the dynamic navigation system Navident (ClaroNav ®Toronto, Ontario Canada)improve the results of our surgeries, letting us to realize a precise Cana sure that we that we considered achievable only with the aid of the computer guided surgery.

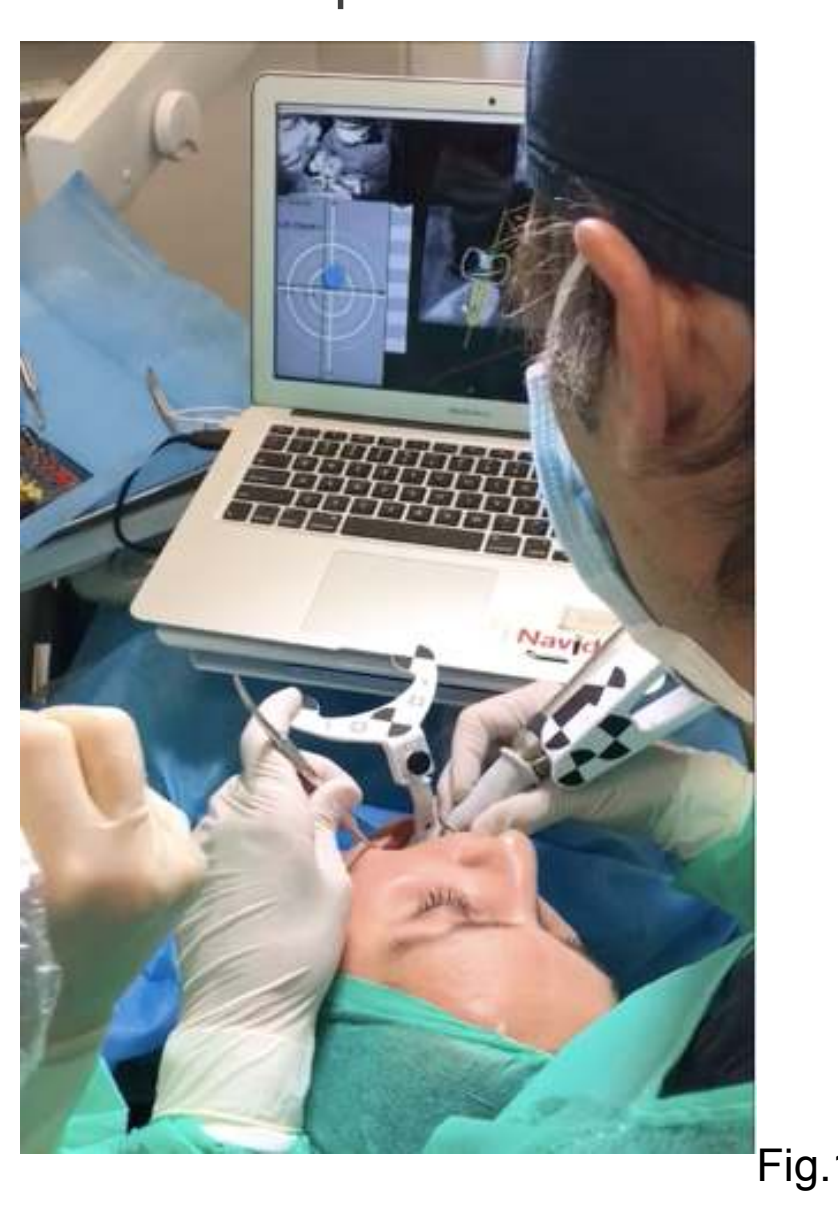

\section{Methods and Materials}

The Navident clinical procedure involves the construction of an individual radiological stent which is accomplish directly in the clinic.For its realization we use a thermoplastic paste that is heated and adapted to the patient's arch to obtain a stable and precise stent.Afterwards a "Ct-marker" is applied to the stent, it will be a reference for the implant software.(Fig.2)

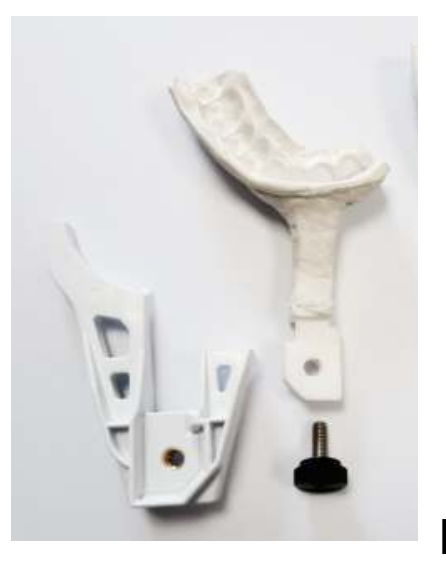

In the planning the implant position follow the prosthetic design trying to have always a central occlusal screw-access. To do a precise prosthetic planification we can combine the CBCT with a digital mock up (Fig.3a-3b). Finished the project we can start with the surgical phase. The patient will wear the radiological stent with a detector that, with the detector put on the surgical handpiece,transmit drills's deepness and 3D position(Fig.1).We can realize the surgery looking at the display of our computer.Using the CBCT like a maps, Navident guides us to center the implant site and to obtain the right angulation.
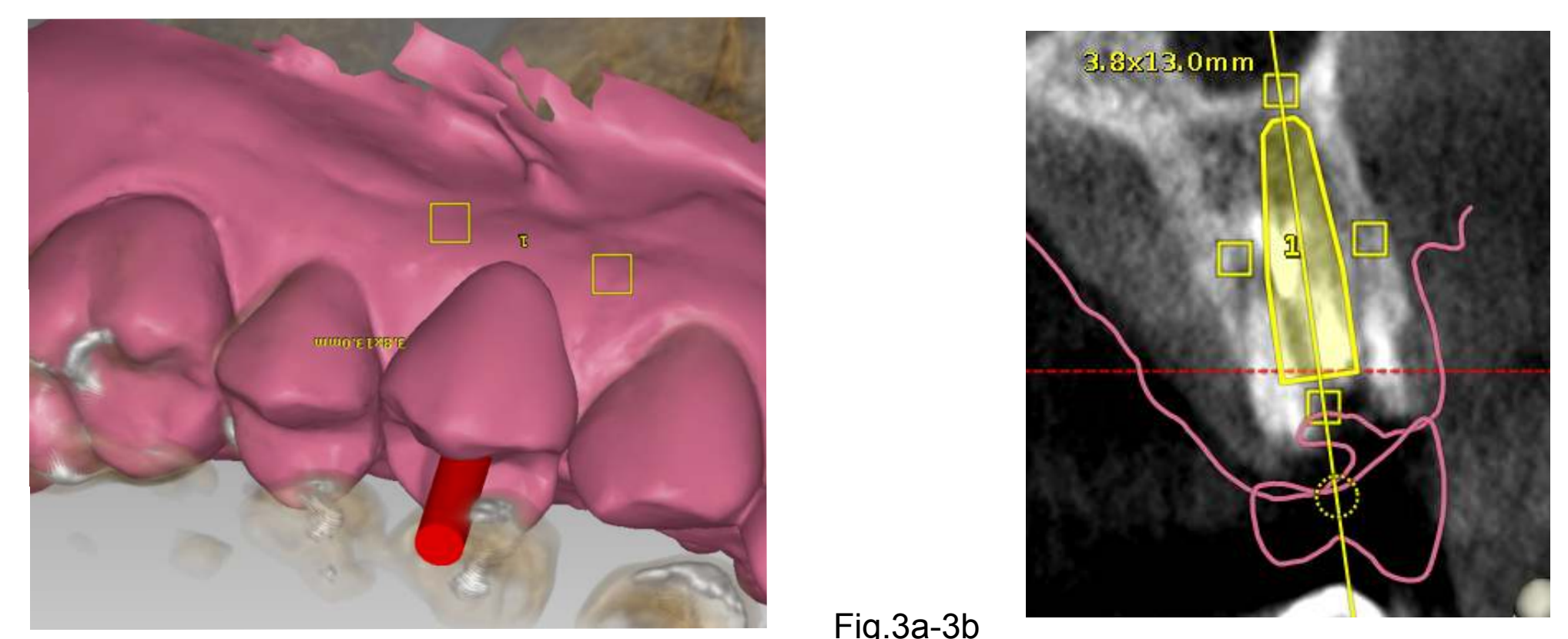

Clinical Case:

Presented below are two clinical case realizing with the methodology previously described.
-Case 1: A 50-years-old- woman was referred to our clinic for the rehabilitation of the partially edentulous area. The patient had been using a removable prosthesis since she was 26 years old.The medical history didn't reveal any systemic diseases. The patient lost her tooth due to periodontal reason.At radiographic examination a severe atrophy was identified in both posterior region of the mandible. After periodontal treatment and long-term maintenance care the patient was ready for the implant surgery. With the aid of Navident we planned the case and we decided to put 7 implants.In the atrophic area we used shorts implants (Way Short Geass ${ }^{\circledR}$, Italy) with a width of $4.5 \mathrm{~mm}$ and a length of $5 \mathrm{~mm}$ and $6.5 \mathrm{~mm}$.(Fig.4a-4b-4c)The incisions were done with a partial thickness to increase the keratinized tissue.
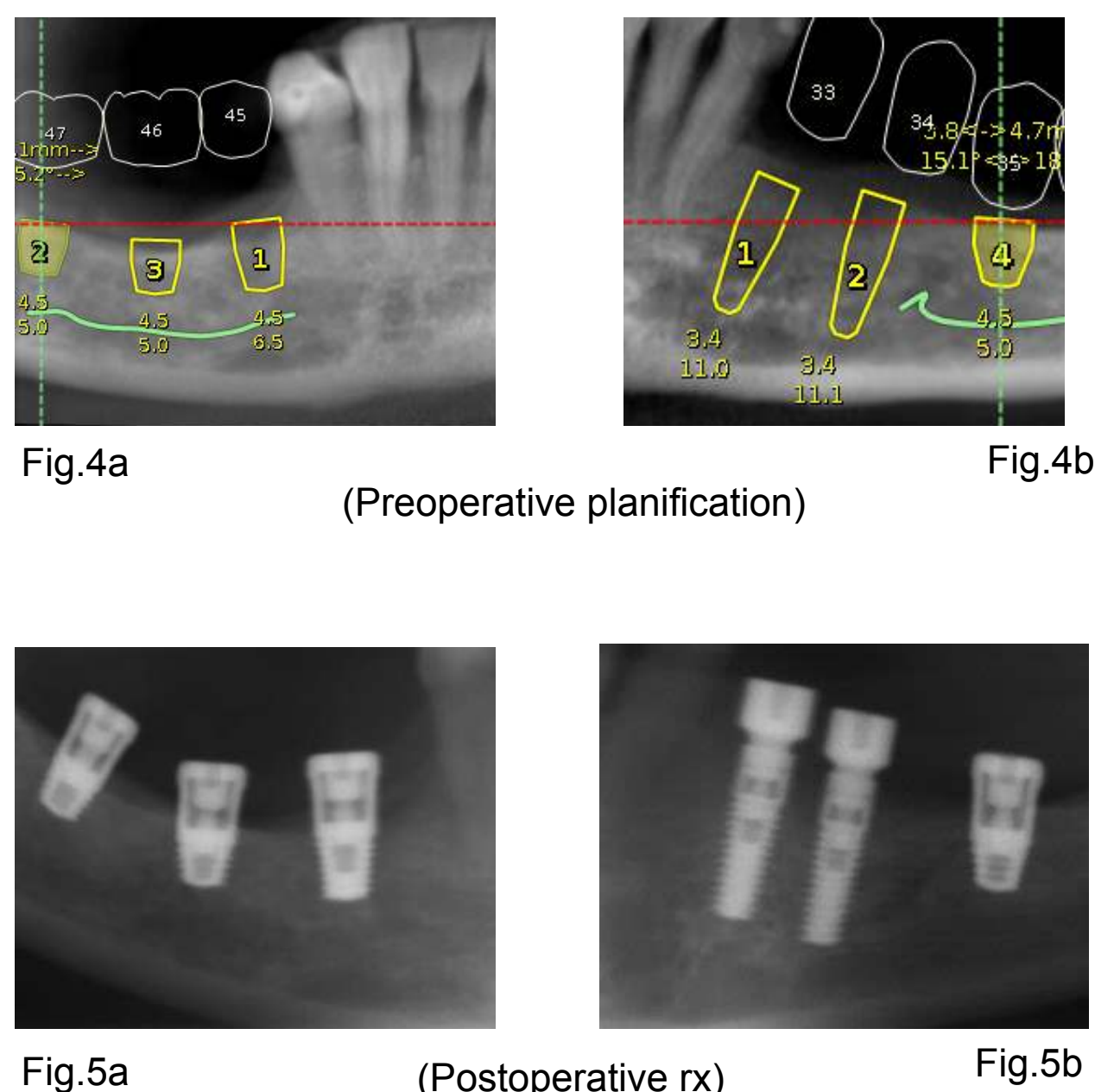

-Case 2: A 58-years-old- woman needed to rehabilitate both posterio region of the mandible.The reason of the tooth loss was decay and failure of endodontic treatment. The medical history did not reveal any systemic diseases. We realize the radiological stent and the CBCT where we appreciate an horizontal bone loss.During the planification we decided to put 5 implant,three of them were short implant (Way Short Geass ${ }^{\circledR}$, Italy) with a width of $4.5 \mathrm{~mm}$ and a length of $6.5 \mathrm{~mm}$. (Fig. $6 a-6 b-6 c)$

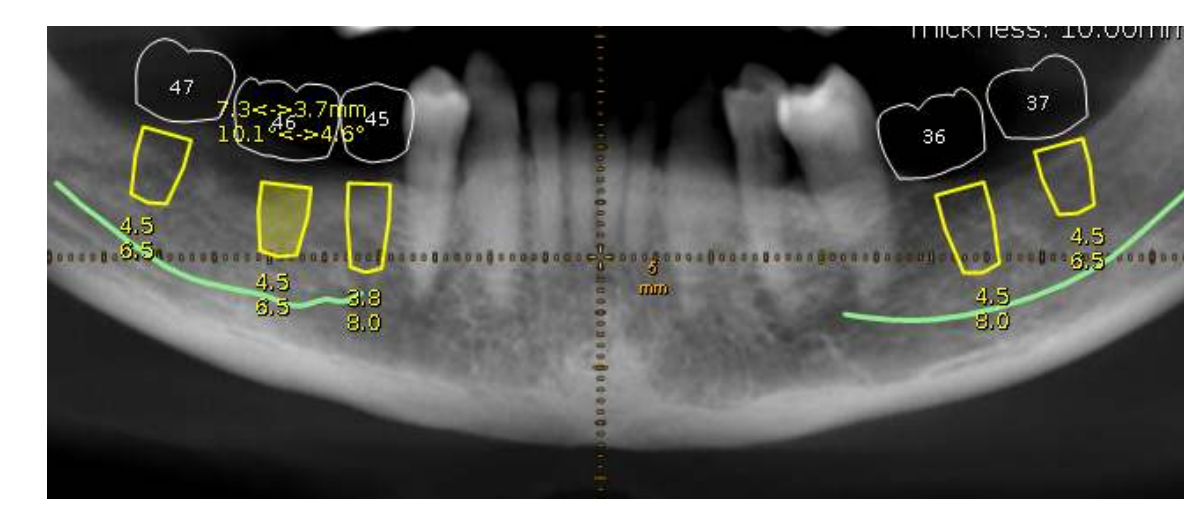

Fig.6a (Preoperative planification)

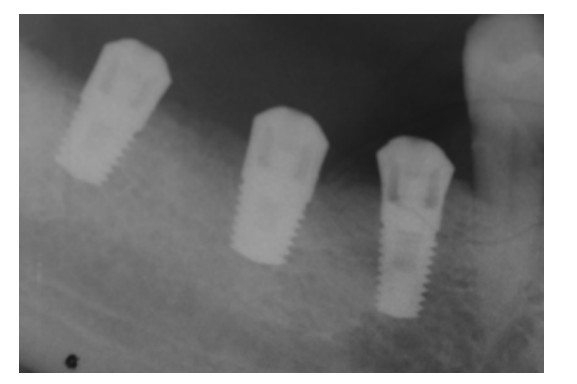

Fig.6b-6c

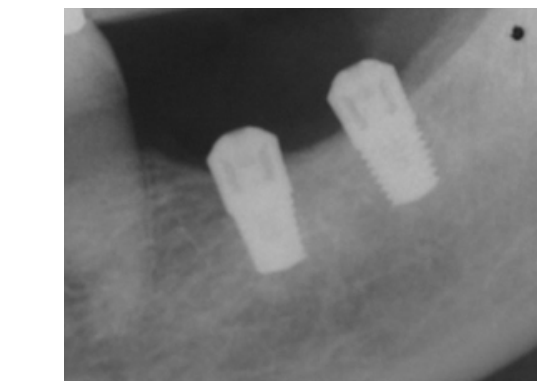

Results

In both cases the implants position was respected like we virtual planned on the software. The anatomic structures, specifically inferior alveolar nerve, were respected and the patients didn't show any complications.

\section{Conclusions}

We consider the dynamic navigation system an important aid for the surgeon because it allows to realize a safety and less invasive surgery, lets realize a correct and precise implants's placement, decreasing human error, improve the ergonomics and in comparison with the traditional computer guided surgery decrease the chair.-time (we only need one visit to plan and make the surgery). We don't need a surgical template and so we can work with patient with a limited mouth's opening and we can manage the tissues doing different types of flaps. With this system we realize a functional and aesthetics rehabilitation.

\section{References}

1. Current state of the art of computer-guided implant surgery,Periodontology 2000

2. Accuracy and complications using computer-designed

stereolithographic surgical guides for oral rehabilitation by means of dental implants: a review of the literature, Clin Implant Dent Relat Res

3. Short implants $(6 \mathrm{~mm})$ vs. vertical bone augmentation and standardlength implants $(\geq 9 \mathrm{~mm})$ in atrophic posterior mandibles: a 5-year retrospective study., Int J Oral Maxillofac Surg.

4. Accuracy of Dynamic Navigation for Dental Implant Placement-ModelBased Evaluation.,J Oral Implantol. 